\title{
Estudo do Índice Biometeorológico Humidex e de sua Correlação com Óbitos em Campinas
}

\author{
João Luís C. De Abreu ${ }^{1}$, Paula D. P. Costa ${ }^{1}$, Ana Maria H. de Avila ${ }^{2}$, Eliana C. de Faria ${ }^{3}$ \\ ${ }^{1}$ Departamento de Controle e Automação (DCA) - Faculdade de Engenharia Elétrica (FEEC) \\ Universidade Estadual de Campinas (UNICAMP) - Campinas, SP - Brasil \\ ${ }^{2}$ Centro de Pesquisas Meteorológicas e Climáticas Aplicadas à Agricultura (CEPAGRI) \\ Universidade Estadual de Campinas (UNICAMP) - Campinas, SP - Brasil \\ ${ }^{3}$ Faculdade de Ciências Médias (FCM) \\ Universidade Estadual de Campinas (UNICAMP) - Campinas, SP - Brasil \\ j175997@dac.unicamp.br, pauladeunicamp.br
}

\begin{abstract}
The present work conducted a regional analysis on the impact of extreme events identified by the biometeorological index Humidex on the deaths from cardiovascular diseases in Campinas. We conducted a comparative analysis of daily death distributions under extreme event versus control-days (MannWhitney U test). Although the literature indicates a correlation between Humidex values and the increase in deaths, we did not found a significant statistical difference.
\end{abstract}

Resumo. Na busca por estratégias de adaptação e pelo desenvolvimento de sistemas de alerta, o presente trabalho conduziu uma análise regional sobre o impacto de eventos extremos apontados pelo índice biometeorológico Humidex, no número de óbitos por doenças cardiovasculares na cidade de Campinas. Para isso, conduziu-se uma análise comparativa de distribuições no número de mortes diárias da cidade, sob evento extremo versus dias-controle (teste $U$ de Mann-Whitney). Apesar da literatura apontar correlação entre valores elevados de Humidex e o aumento de mortes, a análise estatística conduzida não mostrou impacto significativo do Humidex no número de óbitos diários.

\section{Introdução}

Humidex é um índice biometeorológico frequentemente associado a desfechos na saúde. Alguns trabalhos encontraram correlação entre valores extremos de Humidex e uma elevação do número de óbitos na população [Vaneckova et al. 2011, Masterton and Richardson 1979].

O presente trabalho visou investigar evidências dessa correlação na saúde da população de Campinas, São Paulo, Brasil. Mais especificamente, investigou-se se o índice biometeorológico Humidex pode ser associado a um aumento significativo no número de óbitos diários de Campinas, tomando-se como objeto de estudo uma das principais causas de morte no Brasil e no mundo, as doenças do sistema circulatório, associadas ao capítulo IX (Doenças do Sistema Circulatório) da CID (Classificação Estatística Internacional de Doenças e Problemas Relacionados à Saúde) - CID I00-I99. 
Os resultados que serão apresentados buscam complementar e ampliar estudos prévios para o contexto brasileiro, reforçando e contribuindo para o estudo do impacto das mudanças climáticas no sistema de saúde, além de nortear futuros estudos relacionados a diferentes índices de conforto térmico.

\section{Metodologia}

O trabalho integrou e processou duas bases de dados distintas, uma contendo parâmetros meteorológicos, e a outra contendo variáveis de saúde. Os dados de saúde foram obtidos a partir dos registros da Secretaria Municipal de Saúde de Campinas e contemplam informações acerca dos óbitos registrados diariamente no período de 2001 a 2018. Os dados de parâmetros meteorológicos, por sua vez, foram obtidos a partir dos registros da estação meteorológica Centro de Pesquisas Meteorológicas e Climáticas Aplicadas à Agricultura (CEPAGRI) da Unicamp, que contemplam registros diários, a cada 10 minutos, de diferentes parâmetros meteorológicos, no período de 1983 a 2018. O clima de Campinas é caracterizado como um clima subtropical úmido de inverno seco, com elevada umidade relativa e temperaturas amenas.

O Humidex foi computado utilizando-se a Equação 1 . Na equação, $T$ corresponde à temperatura em graus Celsius $\left[{ }^{\circ} \mathrm{C}\right]$ e $e$ à pressão de vapor em hectopascal $[\mathrm{hPa}]$ calculada a partir da umidade relativa do ar.

$$
\text { Humidex }=T+0.5555 \cdot(e-10)
$$

Neste trabalho, adotamos a Tabela 1 como referência para intervalos e faixas interpretativas de valores específicos para este índice, incluindo-se os riscos associados.

Tabela 1. Limites de Humidex [Masterton and Richardson 1979]

\begin{tabular}{|c|l|}
\hline Intervalos de Humidex & \multicolumn{1}{c|}{ Grau de Conforto } \\
\hline $20-29$ & Confortável \\
\hline $30-39$ & Algum Desconforto \\
\hline $40-45$ & Grande Desconforto: Evitar Esforço \\
\hline Maior que 45 & Perigoso: Possibilidade de Insolação \\
\hline
\end{tabular}

Diante dos registros climáticos realizados a cada 10 minutos, houve a necessidade de escolher um valor representativo para o Humidex de cada dia, da série temporal. Tendo em vista o foco do trabalho nos contextos mais críticos e extremos, priorizou-se os valores máximos - dentre os mais de 140 registros diários - como representativos do índice. Além disso, com base na Tabela 1, foram considerados eventos extremos, dias que registraram valores máximos de Humidex superiores a 40.

Com relação às variáveis de saúde, trabalhou-se com os seguintes estratos: gênero (feminino ou masculino), grupo de idade (criança, jovem, adulto ou idoso), raça/cor (branca, preta, amarela, parda ou indígena) e CID-I (dentre as cinco doenças que mais tiveram óbitos registrados - Tabela 2). 
Tabela 2. Cinco doenças com maior número de óbitos registrados

\begin{tabular}{|c|l|c|c|}
\hline CID-I & \multicolumn{1}{|c|}{ Descrição } & $\mathbf{N}^{\circ}$ Óbitos & Percentual \\
\hline I219 & Infarto agudo do miocárdio não especificado & 15290 & $26,27 \%$ \\
\hline I499 & Arritmia cardíaca não especificada & 5310 & $9,12 \%$ \\
\hline I619 & Hemorragia intracerebral não especificada & 3003 & $5,16 \%$ \\
\hline I678 & Outras doenças cerebrovasculares especificadas & 2856 & $4,91 \%$ \\
\hline I64 & $\begin{array}{l}\text { Acidente vascular cerebral, não especificado como } \\
\text { hemorrágico ou isquêmico }\end{array}$ & 2005 & $4,82 \%$ \\
\hline
\end{tabular}

A análise estatística foi conduzida a partir do teste U Mann-Whitney, teste capaz de analisar a significância estatística existente entre dois grupos de amostras independentes, não pareados e que não assumem uma distribuição específica, como é o caso do vetor do número de óbitos. A hipótese nula considerada para esta análise foi a de que o valor de Humidex crítico e o número de óbitos não são variáveis estatisticamente discerníveis.

O nível de significância estatística adotado foi de $5 \%$ e o teste foi aplicado comparando-se duas situações distintas: o número de óbitos em dias com Humidex considerado confortável e o número de óbitos em dias de Humidex crítico - neste caso, valores do índice acima de 40.

\section{Resultados e Discussão}

Após a análise dos resultados, não foi possível obter uma resposta conclusiva acerca do impacto do Humidex no número de óbitos. Como objeto de discussão, toma-se como referência o estrato que apresentou maior significância estatística (menor p-valor) - p-valor igual a 0,00496 - e com o maior número de amostras. Tal estrato consiste nos óbitos do grupo de indivíduos de raça/cor branca com morte atrelada ao CID-I219 referente ao infarto agudo do miocárdio não especificado. Na Tabela 3 , é possível verificar uma descrição mais detalhada do estrato analisado.

Tabela 3. Descrição: óbitos de raça/cor branca com causa atrelada ao CID-I219.

\begin{tabular}{|c|c|c|}
\hline Especificaçóes & Dias de Humidex Confortável & Dias de Humidex Extremo \\
\hline $\mathrm{N}^{\circ}$ Dias com Óbitos & 4397 & 838 \\
\hline $\mathrm{N}^{\circ}$ Dias sem Óbitos & 1117 & 222 \\
\hline Total de Óbitos & 9320 & 1644 \\
\hline Total de Dias & 5514 & 1060 \\
\hline Média de Óbitos Diária & 1,69024 & 1,55094 \\
\hline
\end{tabular}

Com base na descrição e na distribuição dos óbitos para cada estrato, é possível verificar uma ligeira diferença existente entre as médias de óbitos em dias de Humidex confortável e em dias de Humidex extremo. Além disso, nota-se que a diferença nas médias é pequena e que o número de dias sob evento extremo de Humidex é cerca de cinco vezes menor que dias de Humidex confortável. Neste caso, eventuais outliers (eventos raros ou erros de medição) podem impactar mais um dos grupos do que outro. Fenômeno semelhante foi observado nos demais estratos de gênero, idade, raça / cor e CID. 


\section{Figura 1. Distribuição normalizada dos óbitos}

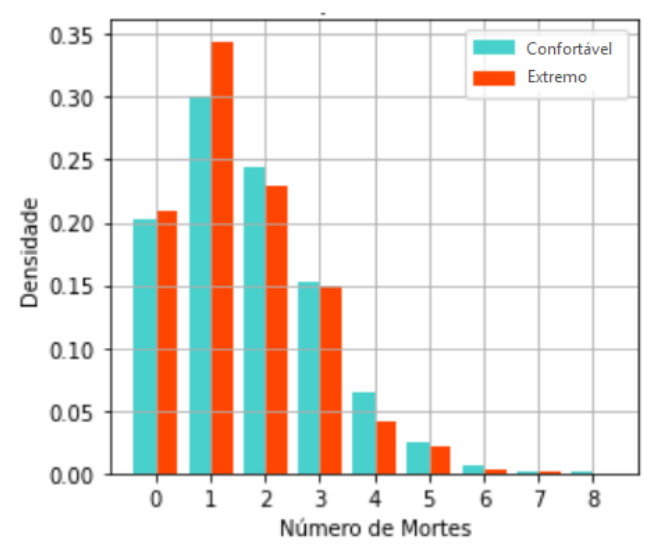

Em resumo, a diferença no tamanho das amostras pode levar a interpretações precipitadas sobre os resultados apresentados. Na Figura 1, por exemplo, é possível visualizar uma distribuição normalizada do tamanho dos vetores de óbitos para os dias considerados confortáveis e para os dias considerados extremos. Neste caso, verifica-se que, apesar das distribuições (Confortável e Extremo) se diferenciarem (a ponto da diferença ser detectada pelo teste estatístico conduzido), à luz do entendimento do problema, não é possível concluir que o Humidex extremo caracteriza um fenômeno de impacto observável.

\section{Conclusão}

Apesar da literatura apontar correlação entre valores elevados de Humidex e o aumento de mortes, a análise estatística conduzida não mostrou impacto significativo do Humidex no número de óbitos diários. Trabalhos futuros incluem o aprimoramento da análise por meio do tratamento de outliers e a adoção da métrica rate ratio. Além disso, é de grande interesse estender essas análises para a base de dados de internações por doenças cardiovasculares em Campinas, assim como introduzir outros índices biometeorológicos.

\section{Agradecimentos}

O presente trabalho foi apoiado pelo programa PIBIC-CNPq e FAPESP Processo 17/20013-1. Os autores também agradecem à Secretaria Municipal de Saúde de Campinas pelos bancos de dados de óbitos e ao CEPAGRI pelo acesso aos dados de sua estação meteorológica.

\section{Referências}

Masterton, J. and Richardson, F. (1979). A method of quantifying human discomfort due to excessive heat and humidity. report cli 1-79. Atmospheric Environment Service, Environment Canada, Downsview.

Vaneckova, P., Neville, G., Tippett, V., Aitken, P., FitzGerald, G., and Tong, S. (2011). Do biometeorological indices improve modeling outcomes of heat-related mortality? Journal of Applied Meteorology and Climatology, 50(6):1165-1176. 